\title{
Brain and odor: II. EEG activity during nose and mouth breathing
}

\author{
TYLER S. LORIG, GARY E. SCHWARTZ, and KATE B. HERMAN \\ Yale University, New Haven, Connecticut \\ and \\ RICHARD D. LANE \\ University Health Sciences/The Chicago Medical School, Chicago, Illinois
}

\begin{abstract}
Previous investigations have suggested that low, undetectable concentrations of odor chemicals alter central nervous system (CNS) activity. Since there can be no odor-free environment, room air also may alter CNS activity. To examine this hypothesis, EEG data were recorded from 20 subjects while they inhaled room air through the mouth or the nose. Results of the experiment indicated that EEG alpha activity in the left hemisphere was reduced for individuals inhaling through the nose, and that EEG beta activity showed greater spatial diversity during nose inhalations. These results demonstrate that room air passing through the nose has a desynchronizing effect upon the EEG and may contain odor information not available through mouth inhalation. These results further substantiate the hypothesis that undetected odors markedly alter ongoing CNS activity.
\end{abstract}

Reception and processing of olfactory information remains poorly understood. The neuroanatomy of the olfactory tract suggests dispersement of olfactory information to widespread and diverse areas of the brain within the first few synapses (Castellucci, 1985). Despite this extensive neural network related to smell, humans are noted for their perceptual and behavioral insensitivity to odors (Doty, 1979; Engen, 1987). Odor memory in subjects without specific odor-related pathology is poor (Eskenazi, Cain, \& Friend, 1986). Habituation to olfactory stimuli is rapid (Carlson, 1981) and may account for humans' inattention to a sustained olfactory environment. Odor thresholds also may differ greatly among normal subjects (Rabin \& Cain, 1986), although thresholds decrease as a function of testing. These findings support the contention that odor is not an important stimulus for the direction of behavior in humans, a contention that seems strange given the importance of odor for nonhuman primates, dogs, and other animals with extensive forebrains (Dewsbury, 1978; Wright, 1982).

One possible explanation for these apparently conflicting findings is that perceptual phenomena related to odor may represent only a fragment of the odor's effects upon the nervous system. Recent evidence from our laboratory (Lorig \& Schwartz, 1988) has indicated that different odors that were perceived as relatively similar produced

Support for this research was provided by International Flavors and Fragrances, Inc., and by NSF Grant BNS-8318984. T. S. Lorig's present address is Department of Psychology, Washington and Lee University, Lexington, VA 24450 . G. E. Schwartz's present address is Department of Psychology, University of Arizona, Tucson, AZ 85721. markedly different patterns of EEG, which had their greatest difference between hemispheres. This finding offers support to the hypothesis that in humans odor perception is only a small part of the neurophysiological activity evoked by odors.

Other findings also support this notion. Recordings from the olfactory bulb indicate increased activity in the presence of undetected odors (Hughes, Hendrix, Wetzel, \& Johnson, 1969). McClintock (1971) determined that components of the odor of urine, probably through their indirect action on the hypothalamus, were important in the regulation of the menstrual cycle of females living in the same setting. Room air, which should not be considered odor free, has also been found to produce EEG alterations in the absence of perceptible odors. Lehmann and Knauss (1976), for instance, found decreased reaction times and increased EEG desynchrony during inhalation of room air. Servit, Kristof, and Kolinova (1977) found that breathing through the nose was more likely to produce epileptiform EEG activity in patients suffering from temporal lobe epilepsy than was mouth breathing, and studies by Werntz and colleagues (e.g., Werntz, Bickford, Bloom, \& Shannahoff-Khalsa, 1983) have demonstrated decreased EEG amplitude over the hemisphere ipsilateral to a single open nostril (unlike some other sensory systems, the olfactory system is predominantly ipsilaterally projecting; Castellucci, 1985). Even though these authors did not suggest that odor information contained in room air may be responsible for these results, all of these findings tend to support the hypothesis that odors present in low, undetected concentrations alter neurophysiological activity. 
To test this hypothesis, subjects in the present experiment were asked to inhale and exhale room air through the nose or mouth. Although it is possible to stimulate olfactory receptors by mouth breathing, the olfactory sensation produced by such breathing is minimal and indicates a greatly reduced receptor pooi (Stevens \& Cain, 1986). If olfactory chemicals in room air stimulate the central nervous system (CNS) to a greater degree during nose than during mouth breathing, such differences should be reflected in the EEG.

\section{METHOD}

\section{Subjects}

Twenty subjects ( 10 male and 10 female) recruited for a personality experiment participated. The subjects were right-handed, ranged in age from 18 to 22 , and reported no history of head injuries.

\section{Materials and Procedure}

The subjects entered the laboratory, were told the nature of the personality experiment, and signed informed consent forms. Goldplated cup-type (10-mm) electrodes were affixed to the scalp at locations F7, F8, T5, and T6 using Elefix electrode paste. Impedances were always below $10 \mathrm{k \Omega}$ and were typically below $5 \mathrm{k \Omega}$. Linked mastoids served as a reference to the active sites. An aneroid bellows was fastened around the chest below the sternum to record the subjects' respirations of room air. EEG and respiration channels were amplified using Grass Instruments preamplifiers (7P5A for EEG, 7P1A for respiration). Recordings were digitized and stored on disk at a sampling rate of $128 \mathrm{~Hz}$ by an IBM-compatible computer, using a Data Translation DT2801-A analog-to-digital converter (12 bit). EEG data were smoothed off-line to a $2-$ to $32-\mathrm{Hz}$ bandwidth, and periods of data containing artifacts were automatically eliminated from records if the wave had a period of greater than $4 \mathrm{~Hz}$.

The subjects were seated comfortably with eyes closed while EEG and respiration data were collected at the beginning of the experiment as a part of the initial set of baseline trials. Two experimental conditions were presented to the subjects in counterbalanced order: inhalation through the nose with exhalation through the mouth, and inhalation through the mouth with exhalation through the mouth. The subjects were instructed to breathe under each condition for approximately $1 \mathrm{~min}$. EEG data were collected throughout the 1 -min period.
Following completion of the experiment, the subjects were debriefed and paid for their participation.

\section{RESULTS AND DISCUSSION}

The raw EEG data for each of the two breathing conditions were separated into inhalation and exhalation groups based upon the aneroid bellows recordings. Onset of inhalation was deemed to have occurred at the point of peak exhalation, and vice versa. The 2 sec of EEG following each inhalation or exhalation were reduced by period analysis to produce measures of the amount of EEG activity in the alpha (8-13 Hz), beta $(13-64 \mathrm{~Hz})$, and theta (4-7 Hz) frequency ranges. Period analysis quantifies the number of waves occurring in each frequency range and has been more fully discussed previously (Lorig, 1986; Lorig \& Schwartz, 1988). Each of the frequency measures was then analyzed using repeated measures analysis of variance (nose/mouth breathing $x$ inhalation/exhalation $\times$ hemisphere $\times$ anterior/posterior).

The respiration conditions produced interesting effects. Theta activity was found to increase in the right hemisphere during exhalation $[F(1,19)=5.87, p=$ .026]. EEG changes of this type have been associated with subjects' self-reports of less tension and anxiety (Lorig \& Schwartz, 1987) and have been reported elsewhere (Lehmann \& Knauss, 1976).

More important to the specific hypothesis under investigation, both alpha activity and beta activity differed as a function of nose or mouth inhalation. Alpha activity changed its hemispheric distribution during nose versus mouth breathing $[F(1,19)=8.20, p=.009]$, showing reduced left hemisphere alpha during nose inhalation and mouth exhalation. Additionally, beta activity showed the greatest anterior/posterior diversity during nose inhalation $[F(1,19)=6.16, p=.023]$. The difference between beta activity in the anterior and posterior regions during nose and mouth breathing is presented in Figure 1. The greatest difference in the distribution of beta activity $O c$ curred during inhalation through the nose.

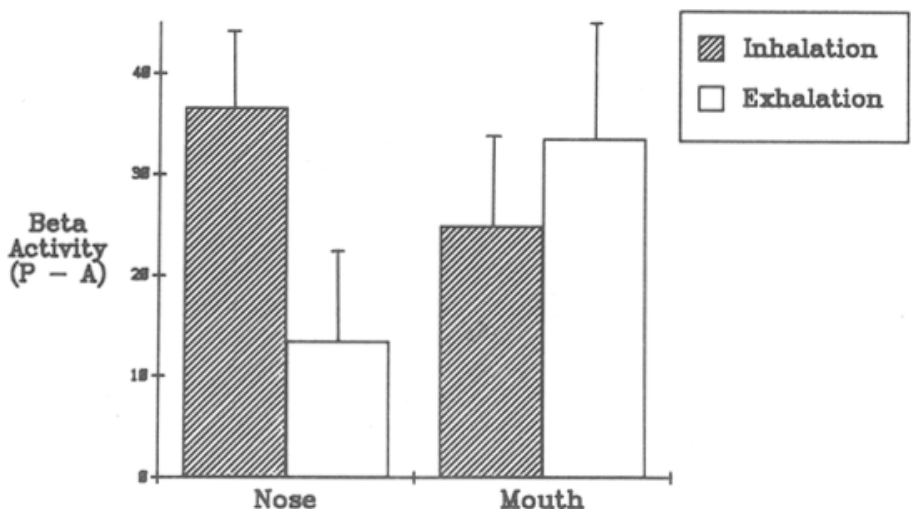

Figure 1. Posterior/anterior distribution (posterior electrode pair minus anterior electrode pair) of beta activity for the four breathing conditions. Error bars indicate average standard errors of the means. 
The results of this study indicate that inhalation of room air through the nose produces a greater alteration in the spatial pattern of EEG alpha and beta activity than does a respiratory mode that provides less olfactory stimulation, such as mouth inhalation. These findings are not easily explained by alternative hypotheses such as tactile stimulation of the nares. Although not conclusive evidence, the data do support the contention that olfactory stimuli exist in low concentrations in room air and stimulate the CNS even in the absence of perceptual phenomena.

These results and those reported earlier (Lorig \& Schwartz, 1988) indicate that odors produce significant alterations in neurophysiological activity in the absence of perceptually different stimuli. This appears to be true for perceptually similar superthreshold odors, as well as for odors that go unnoticed in room air. Brain changes produced by odors may affect a subject's performance or mood, but this relationship would be unrecognized because the stimuli producing these behavioral changes would not be noticed by the subject or the experimenter. Thus, odorous stimuli may have the capacity to modulate CNS activity and behavior in the absence of awareness. Such findings strongly encourage further study of neurophysiological and behavioral effects of odor.

\section{REFERENCES}

CARLSON, N. R. (1981). Physiology of behavior (2nd ed.). Boston: Allyn \& Bacon.

Castellucci, V. F. (1985). The chemical sense: Taste and smell. In E. R. Kandel \& J. H. Schwartz (Eds.), Principles of neural science (2nd ed., pp. 409-428). New York: Elsevier.

DeWsBury, D. (1978). Comparative animal behavior. New York: McGraw-Hill.
DoTY, R. L. (1979). A review of olfactory dysfunction in man. American Joumal of Orolaryngology, 1, 57-79.

ENGEN, T. (1987). Remembering odors and their names. American Scientist, 75, 497-502.

EsKenAzi, B., CAIN, W. S., \& Friend, K. (1986). Exploration of olfactory aptitude. Bulletin of the Psychonomic Society, 24, 203-206.

Hughes, J. R., Hendrix, D. E., Wetzel, N., Johmson, J. W., JR. (1969). Correlation between electrophysiological activity from the human olfactory bulb and the subjective response to odoriferous stimuli. In C. Pfaffmann (Ed.), Olfaction and taste (pp. 172-191). New York: Rockefeller University Press.

Lehmann, D., \& Knauss, T. A. (1976). Respiratory cycle and EEG in man and cat. Electroencephalography \& Clinical Neurophysiology. 40, 187.

LORIG, T. S. (1986). EEG and task performance: A comparison of three analytic techniques. Physiological Psychology, 14, 130-132.

LORIG, T. S., SCHWARTZ, G. E. (1987). Factor analysis of EEG frequencies and self-report. Psychophysiology, 24, 589.

LORIG, T. S., SCHWARTz, G. E. (1988). Brain and odor: I. Alteration of human EEG by odor administration. Psychobiology, 16. 281-284.

McCuntock, M. K. (1971). Menstrual synchrony and suppression. Nature, 229, 244-245.

RABIN, M. D., \& CAIN, W. S. (1986). Determinants of measured offactory sensitivity. Perception \& Psychophysics, 39, 281-286.

Servit, Z., Kristof, M., Kounova, M. (1977). Activation of epileptic electrographic activity in the human by nasal airflow. Physiologia Bohemoslovenica, 26, 499-506.

Stevens, J. C., CAIN, W. S. (1986). Smelling via the mouth: Effect of aging. Perception \& Psychophysics, 40, 142-146.

Werntz, D. A., Bickford, R. G., Bloom, F. E., ShannahoffKHALSA, D. S. (1983). Alternating cerebral hemisphere activity and the lateralization of autonomic nervous function. Human Neurobiology, 2, 39-43.

Wright, R. H. (1982). The sense of smell. Boca Raton, FL: CRC Press.

(Manuscript received March 14, 1988; revision accepted for publication June 15,1988 .) 